\title{
Revisión sistemática: eventos adversos y gestión del talento humano en el contexto hospitalario latinoamericano
}

\author{
Omaira Lucely Araujo-Rosero ${ }^{*}$ orcid.org/0000-0003-4375-5617 \\ Paula Andrea Guerrero-Lasso' ${ }^{1}$ orcid.org/0000-0003-3724-9187 \\ Sonia Maritza Matabanchoy-Tulcán' orcid.org/0000-0002-3681-8440 \\ Claudia Fernanda Bastidas-Jurado' orcid.org/0000-0002-4131-0174
}

A systematic review: adverse events and human talent management in the Latin American hospital context

1. Programa de Psicología, Universidad de Nariño, San Juan de Pasto, Colombia.

Fecha de recepción: Febrero 26 - $2021 \quad$ Fecha de revisión: Septiembre 20 - 2021

Fecha de aceptación: Diciembre 01 - 2021

Gutiérrez-Calderón MA, Díaz-Therán KM. Factores de riesgo psicosocial intralaboral y su relación con dolor músculo esquelético en docentes universitarios. Univ. Salud. Salud. Suplemento1: Especial Psicología y Trabajo. 2021; 23(3):351-365. DOI: https://doi.org/10.22267/rus.212303.249

\section{Resumen}

Introducción: La ocurrencia de eventos adversos (EA) es una problemática de salud pública que impacta el estado de salud del paciente y produce riesgo en el personal sanitario afectando el bienestar laboral y dando lugar a accidentes, enfermedades laborales y acciones inseguras en la atención. Objetivo: Determinar los factores más frecuentes al reportar EA y sus implicaciones para la gestión del talento humano (GTH) en el contexto hospitalario Latinoamericano. Materiales y métodos: Realizado bajo la metodología de revisión sistemática, mediante la búsqueda de artículos publicados entre los años 2009 y 2019, en las bases de datos: BVS, Dialnet, DOAJ, LILACS, MedlinePlus, PubMed, SciELO, ScienceDirect, Redalyc y EBSCOhost. Resultados: Se analizó 41 artículos donde se identificó factores de riesgo y protectores relacionados con la ocurrencia de EA, los cuales se agrupan en tres categorías: referidas al paciente, la organización y al personal sanitario. Conclusiones: Los EA son fenómenos complejos que afectan el sistema de salud. Sus causas se relacionan con la aparición de múltiples factores que tienen que ver tanto con la organización como con el desempeño del personal de salud, inclusive, hasta con el actuar del mismo paciente y su familia.

Palabras clave: Eventos adversos; factores psicosociales; contexto hospitalario; talento humano. (Fuente: DeCS, Bireme).

\section{Abstract}

Introduction: The occurrence of adverse events (AE) is a public health problem that affects patient health status and generates risks for the health personnel, affecting labour wellbeing and leading to accidents, occupational diseases, and unsafe health care procedures. Objective: To determine the most frequent factors when reporting $\mathrm{AE}$ and the implications for the human talent management (HTM) in the Latin American hospital context. Materials and methods: A systematic review was carried out by searching articles published between 2009 and 2019 in the BVS, Dialnet, DOAJ, LILACS, MedlinePlus, PubMed, SciELO, ScienceDirect, Redalyc, and EBSCOhost databases. Results: 41 articles were analyzed, identifying both risk and protective factors related to the occurrence of $\mathrm{AE}$, which were grouped within three categories: related to the patients; to the organization; and to the health personnel. Conclusions: EA are complex phenomena that affect health systems. Their causes are associated with multiple factors that are linked to the organization, the health personnel performance, and the behavior of the patients and their families.

Keywords: Adverse events; psychosocial factor (s); hospital context; human talent. (Source: DeCS, Bireme).

\footnotetext{
*Autor de correspondencia

Omaira Lucely Araujo-Rosero

e-mail: omairalucelyaraujo@gmail.com
} 


\section{Introducción}

El personal sanitario es responsable de velar por el estado de salud de los usuarios, para ello, se debe realizar procesos coordinados, encaminados a favorecer el bienestar y la seguridad de los pacientes, resaltando el rol del trabajador como un recurso de alto valor en los ambientes organizacionales.

Por lo anterior, es necesario fortalecer desde la gestión del talento humano (GTH) la generación de ambientes laborales saludables que aporten a la calidad de vida, propiciando bienestar y disminuyendo factores de riesgo que afecten al personal de salud(1), esto, por medio de estrategias orientadas a la realización tanto personal como profesional, que, a su vez, mitiguen índices como el de rotación, ocurrencia de eventos adversos (EA), accidentalidad y ausentismo entre otros ${ }^{(2)}$. De este modo, la eficiente GTH realizada a partir de procesos coherentes, organizados y articulados aportan al desarrollo humano y organizacional.

La GTH comprende distintos procesos, los cuales representan diversas formas de impactar en las condiciones laborales (3). Así, la adecuada ejecución de cada uno, no sólo representa el correcto funcionamiento organizacional, sino también, el ambiente más propicio para el desarrollo de las funciones articuladas a la satisfacción laboral y el control de los factores de riesgo del ambiente de trabajo.

Caso contrario, las falencias en el diseño del cargo, una inadecuada contratación o la ausencia de procesos de capacitación, desestabiliza al colaborador en el logro de los objetivos institucionales, asimismo, lo expone a diversos factores de riesgo donde el bienestar laboral se ve afectado, incentivando la aparición de enfermedades laborales, accidentes de trabajo, así como también, la ocurrencia de acciones inseguras en la atención en salud(4).

Por otro lado, los factores de riesgos psicosociales, son entendidos como aquellos determinantes que incrementan las probabilidades de sufrir una lesión o enfermedad(5), por su parte, los factores protectores, benefician tanto el desarrollo humano, como el mantenimiento y la recuperación de la salud(1).

En ese sentido, en algunas investigaciones realizadas en el sector salud, se ha identificado que los trabajadores se encuentran expuestos a diversos factores psicosociales, los cuales pueden desencadenar respuestas de estrés y situaciones derivadas como el Síndrome de Burnout, además de alteraciones físicas y psicológicas ${ }^{(6-7)}$.

Aún más, estas investigaciones confirman la condición de vulnerabilidad de esta población, lo anterior, por diferentes aspectos, entre ellos, las jornadas laborales, la sobrecarga de trabajo, las demandas emocionales implicadas en la atención a personas enfermas, el contacto permanente con el sufrimiento, entre otras ${ }^{(8)}$.

De esta manera, los ambientes saludables y la salud de los colaboradores, son condiciones esenciales para la efectividad y el rendimiento de las personas en las organizaciones. Por el contrario, la problemática generada por los factores de riesgo psicosocial, se evidencian tanto en la disminución de la calidad de vida de la población trabajadora, así como también, en el impacto negativo en sus condiciones de salud, al igual que, en los costos que implican para el sistema de seguridad social y para la productividad de las personas y organizaciones ${ }^{(9)}$; provocando, acciones inseguras en medio del cumplimiento de sus funciones, afectando así, la calidad del servicio y la seguridad del paciente(10).

Así las cosas, los EA evidencian la relación entre los factores psicosociales y las actividades desarrolladas por el personal sanitario, las segundas enfocadas a la productividad y calidad de las mismas. Entonces, este tipo de eventos son reconocidos como lesiones o complicaciones involuntarias, las cuales ocurren durante la atención en salud, y que pueden desencadenar en la muerte, la incapacidad o deterioro del estado de salud, entre otras consecuencias, tanto para el paciente como para la organización(11).

Ahora bien, los EA representan un reto importante para los programas de control y prevención, especialmente, por su alta frecuencia, donde su prevalencia ha sido estimada entre el 3\% y el $16 \%$ de los hospitalizados en países de la Organización para la Cooperación y el Desarrollo Económico (OCDE), lo cual está impactando considerablemente la morbilidad y mortalidad de la población ${ }^{(12) .}$

Así, los EA son catalogados en dos tipos: los primeros, denominados prevenibles, que se pudieron haber evitado mediante el cumplimiento de los estándares 
del cuidado asistencial, y los segundos, los no prevenibles, que se presentan a pesar del cumplimiento de dichos estándares(13).

Pues bien, con el propósito de evitar los posibles perjuicios resultantes de los EA, y así disminuir el número de fallos en la atención prestada en los servicios sanitarios, las instituciones de salud incorporan la Cultura de Seguridad del Paciente (CSP) para ofrecer el mejor servicio posible, sin generar perjuicios al paciente, ni más gastos al sistema de salud. Dentro de esta práctica es relevante el reporte de los EA, ya que permiten identificar, analizar y prevenir futuros fallos(14).

Es así como, en Latinoamérica, al observar el panorama de la seguridad del paciente, se identifica en algunas investigaciones, que una de las causales de los EA está relacionada con las ocurrencias del error humano $(\mathrm{EH})^{(15-16)}$, es decir, eventos que han generado daño de manera innecesaria al paciente(17); asimismo, están relacionados con malas o descuidadas prácticas de los profesionales en salud por la falta de adherencia a protocolos, inadecuado manejo de los niveles de estrés y excesiva carga de trabajo, lo cual disminuye la calidad de la atención hacia el paciente, y por tanto, es un factor de riesgo que incrementa la ocurrencia de EA.

Es por esto, que el presente trabajo investigativo busca dar respuesta a la pregunta ¿Cuáles son los factores más frecuentes al reportar EA y las implicaciones de la GTH en el contexto hospitalario latinoamericano? Que, a su vez, establece el objetivo de determinar los factores más frecuentes al reportar EA y las implicaciones de la GTH en el contexto hospitalario latinoamericano.

\section{Materiales y métodos}

\section{Tipo de estudio}

Se realizó una revisión sistemática de los factores más frecuentes al reportar EA y la implicación de la GTH en el contexto hospitalario latinoamericano(18).

\section{Unidad de análisis}

Se analizaron 41 estudios (originales primarios, estudios empíricos y revisiones de la literatura), realizados y publicados en América Latina entre los años 2009 y 2019, los cuales poseen como tema central los factores relacionados con los EA en el contexto hospitalario y la implicación de la GTH.

\section{Bases de datos}

Se trabajó en la revisión de artículos pertenecientes a bases de datos de libre acceso tales como: Biblioteca Virtual de Salud (BVS), Dialnet, Directory of Open Access Journals (DOAJ), Literatura Latinoamericana y del Caribe en Ciencias de la Salud (LILACS), Medline Plus, PubMed, SciELO, ScienceDirect, y Red de Revistas Científicas de América Latina y el Caribe, España y Portugal (Redalyc); además, de EBSCOhost como la una única base de datos paga, por cuanto la casa de estudios del equipo investigador tiene acceso a ella.

Adicionalmente, los estudios se identificaron a partir de los parámetros de búsqueda, para este caso, la combinación de palabras clave como: eventos adversos, contexto hospitalario o de salud, error humano y talento humano, las cuales fueron validadas en diferentes tesauros; cabe mencionar que la búsqueda también se realizó en el idioma portugués.

\section{Criterios de inclusión}

Se tuvieron en cuenta artículos publicados en las bases de datos referenciadas anteriormente, que, además, en su título, resumen o palabras clave, Contaran con al menos dos de los términos de búsqueda: factor(es), error humano, eventos adversos, contexto hospitalario y Latinoamérica; asimismo, que fueran textos completos, publicados entre los años 2009 y 2019, en los idiomas español y portugués, con población, producción y publicación únicamente de países de América Latina.

\section{Criterios de exclusión}

Se excluyeron artículos duplicados, excesivamente específicos en un área de la salud, que no correspondían a investigaciones empíricas, o que sus resultados o análisis no giraban en torno a los factores más frecuentes en el reporte de EA y la implicación de la GTH en el contexto hospitalario latinoamericano. 


\section{Evaluación de la calidad de los trabajos}

Los artículos seleccionados fueron aquellos presentes a texto completo, que, además, con criterios de autenticidad, transferibilidad y auditabilidad; así mismo, que hayan sido publicados en revistas latinoamericanas, teniendo en cuenta los criterios de inclusión y exclusión para cada uno de ellos. De igual forma, cada artículo abordó la temática propuesta en Latinoamérica para su respectivo estudio.

\section{Instrumentos o técnicas}

Se utilizó una matriz de registro documental basada en el protocolo PRISMA-P(19), la cual se diligenció tras la revisión detallada de cada uno de los artículos, y asimismo, permitió la consignación de las siguientes categorías: título original del artículo, autores, año de publicación, referencia y URL y/o DOI de publicación, revista donde se publicó, resumen, región del estudio, método (tipo de estudio y participantes), resultados principales, factores (de riesgo y protectores), conclusiones generales y concepto de evento adverso.

\section{Plan de análisis de información}

Los datos recolectados sobre los artículos finales fueron consignados en una matriz en el programa Excel, esta, contenía las categorías y subcategorías de análisis de los mismos, siguiendo el protocolo PRISMA-P, posteriormente, se realizó su análisis.

\section{Consideraciones éticas}

El estudio no representa ningún riesgo, ya que no se presenta contacto directo con seres humanos, además, se emplearon técnicas y métodos de investigación documental retrospectiva, con citas a las respectivas fuentes y salvaguardando los contenidos e ideas originales de los autores, lo anterior, en coherencia con los criterios éticos expresos en la Resolución 8430 de 1993 por la cual el Ministerio de Salud de la República de Colombia establecen las normas científicas, técnicas y administrativas para la investigación en salud.

\section{Resultados}

Se identificaron 1.208 artículos, de los cuales, se aceptaron 41 estudios para la presente revisión sistemática. Las fases para la selección de artículos se evidencian en la Figura 1.

Del número final de artículos, se pudo identificar que el $56 \%(n=23)$ fue escrito en el idioma español, mientras que el $44 \%(n=18)$ en idioma portugués. En cuanto al país de procedencia de los artículos, se

\section{Número total de articulos encontrados

$$
=1.208
$$

BVS (160), Dialnet (246), Doaj (76),

LILACS (35), Medline Plus (44),

PubMed (3), Redalyc (490), SciElo (71),

ScienceDirect (20), EBSCOhost (63)

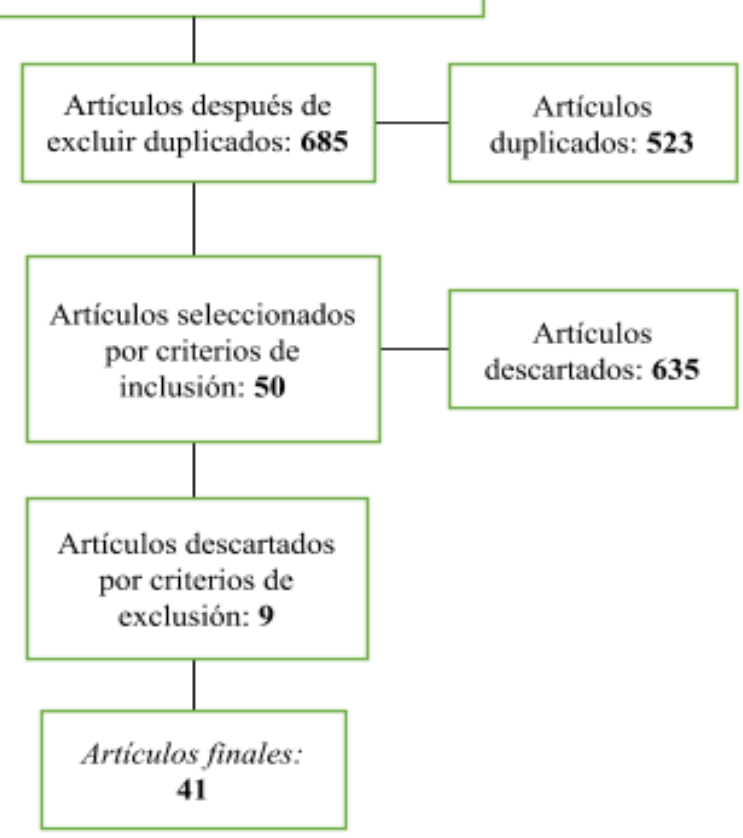

Figura 1. Fases de la selección de artículos

estableció que Brasil es el lugar donde más manuscritos se encontraron con un 43,9\% ( $n=18)$, seguido por México 19,5\% (n=8), Colombia 17,0\% $(n=7)$, Uruguay 4,8\% (n=2), Chile 2,4\% (n=1), Ecuador 2,4\% (n=1), Argentina 2,4\% $(n=1)$, Costa Rica 2,4\% (n=1), México, Argentina y Colombia $2,4 \%(n=1)$ y Argentina, Brasil, Chile, Colombia, Cuba, México y Perú 2,4\% (n=1).

Las características generales de los estudios seleccionados se muestran en la Tabla 1 . Por otra parte, siete artículos no incluyeron una definición de EA.

Un análisis de los artículos restantes permite distinguir las siguientes categorías:

1. Acciones emitidas. Los EA son entendidos como acciones inseguras, situaciones inesperadas o impredecibles, complicaciones, incidentes, errores, ocurrencia o evento clínico indeseable, fallas en la planificación de la atención, presencia de complicaciones indeseables o circunstancias 
que producen daño, atribuibles a la atención en salud $y$ no a la enfermedad del paciente $(12,15,17,19,20-26,27)$.

2. Intencionalidad de los actos. El daño o lesión causada durante la atención en salud es involuntario, no intencionado e innecesario y que adicionalmente, puede ser prevenible o no prevenible ${ }^{(12,24,28-30)}$

3. Consecuencias hacia el paciente. Una estancia hospitalaria prolongada, deterioro del estado de salud, afectación física, psicológica y social, sufrimiento, incapacidad, discapacidad y muerte(17). Para la institución de salud: incremento en los costos a causa de la estancia hospitalaria prolongada, monitorización e intervenciones adicionales y situaciones jurídicas. Para el personal de salud: afectación psicológica(19,23,25,27,32,33,34).
4. Grado de gravedad e impacto. Los EA son clasificables de acuerdo con el grado de gravedad: grave y no grave; y de acuerdo al impacto del daño: cuasi error, incidente sin daño e incidente con daño(19,28,23).

En cuanto a los factores de riesgo relacionados con la ocurrencia de EA, se identificaron tres categorías correspondientes a factores de riesgo del paciente, factores de riesgo relacionados con la organización y factores de riesgo relacionados con el personal de salud, tal como se muestra en la Tabla 2.

Del total de artículos analizados, dieciocho de ellos mencionaron factores protectores organizados en dos categorías: referidos a la organización y hacia el personal de salud, tal como se muestra en la Tabla 3.

Tabla 1. Características de los estudios

\begin{tabular}{llr}
\hline & Característica & Porcentaje y número de estudios \\
\hline Participantes o unidad de análisis & Población & $56 \%(\mathrm{n}=23)$ \\
& $\begin{array}{l}\text { Documentos: historias clínicas, reportes de } \\
\text { eventos adversos, artículos }\end{array}$ & $44 \%(\mathrm{n}=18)$ \\
\hline Sector al que pertenece la & Público & $59 \%(\mathrm{n}=24)$ \\
institución donde se realizó el & Privado & $7 \%(\mathrm{n}=3)$ \\
estudio & No especifica & $34 \%(\mathrm{n}=14)$ \\
\hline Metodología (tipo de estudio) & Descriptivos & $71 \%(\mathrm{n}=29)$ \\
& Analíticos & $19 \%(\mathrm{n}=8)$ \\
& Experimentales & $10 \%(\mathrm{n}=4)$ \\
\hline Sector de aplicación del estudio & Urbano & $78 \%(\mathrm{n}=32)$ \\
& No especifica & $22 \%(\mathrm{n}=9)$ \\
\hline
\end{tabular}

Tabla 2. Factores de riesgo

\begin{tabular}{|c|c|}
\hline Categoría & Factores de riesgo identificados \\
\hline Paciente & $\begin{array}{l}\text { - Género: masculino(24,27,32). } \\
\text { - } \quad \text { Edad: lactantes, preescolares (de } 0 \text { a } 5 \text { años) o superior a los } 45 \text { años(23,24,25,27,32,33,35). } \\
\text { Variables clínicas: estado de conciencia del paciente (riesgoso/inquieto, poco cooperativo/incumplido o } \\
\text { con intento de autodaño), padecer enfermedades como hipertensión arterial, diabetes, hipoalbuminemia y } \\
\text { obesidad; complejidad de la condición clínica del paciente }(17,19,23,27,35) \text {. }\end{array}$ \\
\hline Organización & $\begin{array}{l}\text { - Infraestructura: lugar donde se brinda el servicio inadecuado o defectuoso o viejo, insumos inadecuados } \\
\text { con lo que se brinda el servicio, insuficientes, defectuosos o viejos, hacinamiento de pacientes }(31,36,37,38) \text {. } \\
\text { - Proceso de selección: debilidades en la formación técnica y profesional del personal de salud, indefinición } \\
\text { de funciones laborales(11,26,33,39,40). } \\
\text { - Contratación: falta de personal en las diferentes áreas y alta rotación del personal }(11,26,30,31,36,40) \text {. } \\
\text { - Proceso de capacitación: bajos niveles de educación continua, estandarización de políticas, protocolos y } \\
\text { procedimientos, falta de capacitación para casos específicos, guías clínicas desactualizadas o } \\
\text { incompletas }(11,28,29,33,37) \text {. } \\
\text { - Seguimiento: falta de control de las tareas y supervisión, mala conducción del personal, medidas punitivas } \\
\text { ante el reporte de eventos adversos(26,27,31,35,37,39). }\end{array}$ \\
\hline
\end{tabular}


- $\quad$ Carga de trabajo excesiva: dobles turnos, multiplicidad de pacientes asignados, ignorar la importancia de la medición de la carga de trabajo $(11,17,24,26,27,30,31,33,35,36,37,40,41,42)$.

- Multiplicidad de servicios: tipo de servicio (complejidad) $(15,27,28)$.

Personal de salud • Administración de medicamentos: confusiones en la administración de medicamentos, errores en la dosis o vía de administración, fallas en la continuidad de la prescripción y en la preparación y/o de los fármacos(15,17,30,34,36,42,43).

- Sobrecarga laboral: ausencia de descansos debido a múltiples contratos laborales, así como la fatiga, estrés e insatisfacción con las condiciones de trabajo(26,27,36,38,39-42).

- Errores humanos: faltas relacionadas con la actividad o actos inseguros como omisión, distracción, errores, fallos de atención, incumplimiento de procedimiento, presión por el tiempo, preocupación, aburrimiento, comportamientos y actitudes insatisfactorias(11,25-28,35,36,41,42,43).

- Falta de adhesión a protocolos: déficit en el cumplimiento de las normas y rutinas institucionales, el déficit en la supervisión de enfermería y la inexperiencia profesional, la falta de información previa sobre la salud de los pacientes, ausencia de lavado de manos, utilización incorrecta de los estándares del cuidado asistencial(12,26-29,30,31,35,40,42,44,45).

- Ausencia de reporte de EA: baja adherencia al registro de notificaciones de eventos adversos, documentación inadecuada o la ausencia de información en el registro médico, represalias del equipo médico por alertar posibles problemas o EA(12,17,23,26-29,35,37,40,46,47,48).

- Competencias: la falta de habilidad y competencia, problemas de comunicación y formación y entrenamiento inadecuado, falta de autonomía del líder, la necesidad de decisiones urgentes de alto riesgo, la variabilidad en la formación de los profesionales médicos y enfermeras, inexperiencia(11,12,17,2529,30,31,36,40,41,43,44,45,47-50).

- Fallas en el diligenciamiento de la historia clínica: subestimación de la verdadera magnitud de estos eventos, eventos no registrados de la forma correcta $(17,29,40,42,44,47,48)$.

Tabla 3. Factores protectores

\begin{tabular}{|c|c|}
\hline Categoría & Factores protector identificados \\
\hline Organización & 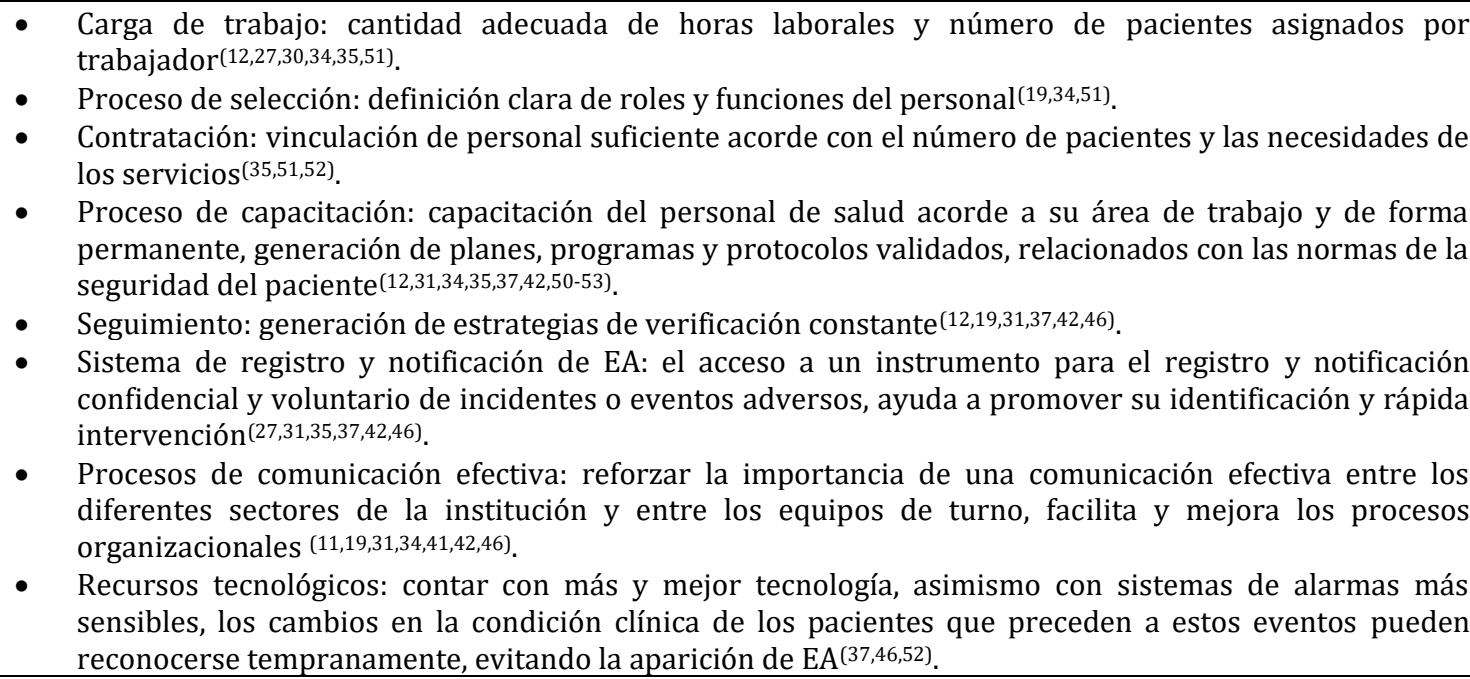 \\
\hline Personal de Salud & $\begin{array}{l}\text { - Notificación de EA: la notificación de errores tiene la intención de reducirlos }(27,31,35,37,46) . \\
\text { - } \quad \text { Profesionalismo: la conducta del profesional, atenta, empática(19,41). } \\
\text { - } \quad \text { Trabajo en equipo: contribuir a la buena convivencia laboral, generación de espacios amenos en el trabajo } \\
\text { multidisciplinar( }(11,19,31,34,41,46) \text {. }\end{array}$ \\
\hline
\end{tabular}




\section{Discusión}

De acuerdo a los hallazgos encontrados y tras el análisis de la información, sobre la definición de EA, se tuvo en cuenta diferentes categorías, tales como las acciones emitidas, la intencionalidad de las mismas, las consecuencias sobre el estado de salud del paciente, el grado de gravedad y el impacto del daño producido.

De acuerdo con lo anterior, en conjunto con el análisis de los resultados, se puede identificar que las definiciones de EA retoman los daños o consecuencias hacia el estado de salud del paciente, y en algunos casos, los sobrecostos que representa para la institución de salud; información que guarda relación con dos revisiones sistemáticas $(48,54)$ en las cuales, la definición de EA gira en torno a términos como daño no intencional ocasionado al paciente, atribuible al cuidado médico más que a la enfermedad, lo que indica cierta universalización del término en la literatura concerniente con la salud.

Relacionado con lo anteriormente expuesto, la teoría revisada no profundiza sobre las consecuencias de los EA en las segundas víctimas, quienes se consideran como todo profesional proveedor de servicios sanitarios, que participa en un EA, un error médico y/o una lesión relacionada con el paciente, no esperada y que se convierte en víctima en el sentido de que queda emocional y profesionalmente traumatizado por el suceso. Derivado de lo anterior, se presentan consecuencias a nivel emocional, tales como respuestas de ansiedad, despersonalización, frustración, culpa, síntomas depresivos, insomnio o dificultades para disfrutar sueño reparador, reexperimentar el suceso una y otra vez, ira y cambios de humor en casa(55).

Por otra parte, el impacto a nivel profesional, se evidencia en la presencia de preocupación mórbida por su rendimiento y capacidad profesional, lo cual llega a afectar su toma de decisiones clínicas, obnubilación, confusión y dificultades para concentrarse en una tarea. Uno de los estudios menciona la presencia de miedo a consecuencias legales, asimismo, temor a la pérdida de reputación profesional a raíz de la notificación de EA(47).

De alguna manera, se reconoce que tras un EA se incrementa la posibilidad de que ocurra otro, precisamente por la afectación emocional y el impacto a nivel profesional(56). En este sentido, se considera importante que, al momento de estudiar los EA, se incluya a las segundas víctimas para lograr un entendimiento global del suceso y formular acciones interventivas integrales.

Aún más, la ocurrencia de EA se presenta en mayor medida en pacientes de género masculino, en concordancia con los resultados de una investigación( ${ }^{(56)}$ donde se destaca que este género experimentó un mayor número de EA, así también lo afirma una revisión de la literatura frente a $\mathrm{EA}^{(58)}$. Por el contrario, otra investigación reporta como resultado una mayor prevalencia de EA en mujeres(58).

Adicionalmente, los hallazgos muestran que la edad es una variable que predispone la aparición de EA, por cuanto, la infancia y la longevidad, son los extremos en los cuales hay mayor ocurrencia de los mismos, hallazgo que coincide con una investigación que menciona que los primeros EA que se enfrentan pueden datar desde la infancia y niñez temprana, además, estos suelen ser los más nocivos ${ }^{(59)}$.

En complemento de lo anterior, pese a que la etapa infantil ha sido comparativamente desatendida en términos investigativos, ha llegado a estimarse que más del $60 \%$ de los niños en este rango de edad han vivido situaciones adversas, potencialmente perniciosas. Por su parte, algunos estudios sugieren que los mayores de 60 años sufren más EA, lo anterior a razón de sus comorbilidades, lo cual incrementa su vulnerabilidad(60,61,62).

Ahora bien, en cuanto a algunas variables clínicas, el análisis de la información identificó que el estado de conciencia, la presencia de enfermedades crónicas y complejidad del estado de salud del paciente, son factores importantes que se relacionan con la aparición de EA, aspectos que guardan relación con investigaciones y otras revisiones sistemáticas, donde se destaca que la mayoría de EA se presentan en personas con enfermedades crónicas agudizadas, con estados de conciencia disminuido, y cuyo estado de salud es complejo, por cuanto puede cambiar en cualquier momento(57,62).

Además, otras investigaciones refieren que los EA han sido vinculados a un peor estado de salud, producto de condiciones médicas crónicas, asma, obesidad, disfunción miccional, mala salud dental, mayor presencia de caries, menor calidad de vida 
relacionada con la salud y más preocupaciones de salud y atención médica(57,59).

Por otra parte, haciendo alusión a los resultados de la presente investigación, frente a la mayor vulnerabilidad de la ocurrencia de EA en pacientes cuyas edades se encuentren en los extremos de la vida (infantes y adultos mayores), la teoría menciona que esta población requiere cuidado de un familiar o acompañante, quien también debe velar por la evitabilidad de EA, y no ser una tarea exclusiva del personal de salud(57,59).

En coherencia con lo anterior, se menciona que debe existir apoyo asistencial adicional para grupos vulnerables como: ancianos, enfermos graves o en estado terminal y niños, el cual puede ser brindado por un cuidador adicional o su propia familia(63).

Asimismo, en lo referente al autocuidado por parte del paciente frente a su salud e integridad, o por parte de su familia y/o cuidador, se identifica que el paciente y su familia o cuidador tienen un papel preponderante, como parte de los actores que pueden apoyar a la evitabilidad de EA, así lo concluye una investigación donde menciona que el paciente y su familia, pueden asumir el autocuidado de su seguridad, en lo que a EA e infecciones se refiere(64).

De este modo, para lograr que se interesen y se involucren en el autocuidado, es necesario informarles sobre cuál es su responsabilidad en ello, asimismo, los aspectos que los prestadores de servicios deben cuidar. En esta investigación, se establece que entre los principales EA se encuentra la caída de los pacientes, cuestión relacionada no sólo con el cuidado de enfermería, sino también, con el acompañamiento por parte del familiar o cuidador(27).

Así las cosas, en Colombia algunas instituciones de salud trabajan en la implementación y fortalecimiento de la política de seguridad del paciente, ante esto, una entidad de salud menciona que la coparticipación de los pacientes en su autocuidado, es una estrategia para garantizar atención segura y disminuir la posibilidad de EA(65).

De igual modo, es importante mencionar que se encontraron factores de riesgo asociados a la aparición de los EA generados por la organización y el personal de salud, es así que, se considera que los EA que tienen como causa los recursos o la gestión organizacional, los cuales pueden presentarse por adaptación de la gestión de la carga asistencial, disponibilidad de camas o servicios, idoneidad de recursos humanos y materiales, correcta organización de equipos, disponibilidad y adecuación de protocolos, políticas, procedimientos y directrices, así como también, los generados por el personal de salud, tales como incidentes en relación con los procedimientos clínicos vinculados con los cuidados hospitalarios y relativos al proceso de comunicación, generan un daño imprevisto e inesperado(66).

Además, entre los daños identificados están aquellos que dan lugar a una hospitalización prolongada, una discapacidad en el momento del alta médica, o ambas cosas, conjuntamente, con pérdidas en la enorme cantidad de recursos económicos, asimismo, promoviendo el detrimento de credibilidad del sistema de salud en su conjunto(1,67).

Por otra parte, entre los factores de la organización se encuentran los recursos físicos, como la infraestructura, comprendiéndose como una condición del ambiente que predisponen una acción insegura. En este sentido, al presentarse deficiencias en las condiciones estructurales en la entidad, también se dificulta el cumplimiento de protocolos(68).

En adición a lo expuesto con respecto al recurso físico, este es un aspecto que deja visualizar la necesidad de modernización en salud, dado que, este aspecto estructural es un elemento fundamental para la prestación de servicios, permite lograr un acceso oportuno a las instalaciones físicas, incentiva modelos de desarrollo sostenible, permite contar con ventajas competitivas, y garantiza de alguna manera, el suministro de una atención eficiente en salud(69).

Ahora, en lo concerniente al personal sanitario, se encuentran procesos relacionados con la complejidad del tipo de servicio prestado, asimismo, la sobrecarga laboral, la cual imposibilita al trabajador el uso adecuado de descansos y pausas, lo que genera estrés y fatiga, es así que, las condiciones laborales particulares de cada servicio de atención, junto con el nivel profesional y la duración de la jornada laboral, aparecen como importantes fuentes de carga mental(70).

Lo anterior, se vincula a la existencia de distintos niveles de fatiga, situación que aumenta la probabilidad de desatar errores humanos, los mismo que llevan al desarrollo de actos inseguros o la 
violación de procedimientos(71). Las jornadas extensas y la rotación de turnos, generan repercusiones sobre la vida, la salud y el bienestar de los pacientes, es así que se favorece la presencia de los $\mathrm{EA}^{(72) .}$

De igual modo, es posible identificar que algunos estudios mencionan que la sobrecarga laboral es la variable que más influye en el error, dado que las cargas de trabajo excesivas van provocando progresivamente en el profesional, cansancio, bajo rendimiento, fatiga, y todo ello, contribuye al aumento de riesgo de cometer errores(73).

Por lo anterior, entre los factores protectores para la prevención de los eventos adversos dentro de la organización, se encontró la posibilidad de llevar un sistema de registro y notificación de EA, el cual, facilita la identificación e intervención oportuna, logrando con ello un sistema de vigilancia $y$ notificación de incidentes de EA, que facilite la detección temprana de los mismos(73).

Al respecto, cabe aclarar que los sistemas de vigilancia y notificación también contribuyen a la prevención de otros sucesos adversos, dado que permiten conocer los factores que favorecen la aparición, y establecer acciones preventivas ${ }^{(74)}$, por ello, la notificación de los EA, es un instrumento esencial en la gestión de la atención(27,74), además, permite a los profesionales asistenciales, compartir responsabilidades con los administradores, para con ello, estimular acciones correctivas, y así, evitar la repetición de los errores y prevenir futuros ${ }^{(75)}$.

En otro sentido, para el personal sanitario se identificó que la notificación de los EA, la comunicación efectiva, el profesionalismo y la formación aportan en la reducción de los EA(48). Así, se ha observado una relación directa, entre una comunicación clara y efectiva con la atención médica de alta calidad, que, a su vez, garantiza mayor seguridad para el paciente.

Como complemento, en lo que respecta al profesionalismo y formación recibida por el personal de salud, se considera fundamental que las instituciones prestadoras de servicio, busquen profesionales con una formación idónea y se interesen en la continuidad de dicha formación, en la cual prime la responsabilidad en el acto de cuidar(71).
Asimismo, se encontró documentos que mencionan la importancia de la comunicación entre los profesionales de la salud y el paciente, ya que, en esta, se genera una interacción que alienta y valora al personal, además, da mayor confianza al usuario(76); lo anterior, favorece la transformación del escenario en las instituciones de salud, mejorar la cultura de seguridad, y disminuye la posibilidad de errores relacionados con la asistencia(12,77).

Paradójicamente, se encuentra en esta investigación que respecto a la función del talento humano confluyen dos miradas, tener el potencial de ser factores de riesgo o factores protectores según la gestión que se haga, es así que, es fundamental una buena GTH, dado que esta influye de manera directa en una adecuada cultura de seguridad del paciente, aportando en el modo de actuar del trabajador, que permite prevenir la presencia de EA(78).

En coherencia, se reconoce que la función del talento humano es punto fundamental dentro de las instituciones hospitalarias, que al no cumplir con su propósito, puede provocar no sólo el fracaso para la excelencia de la calidad, sino también, para el cuidado del paciente(79).

En sentido adicional, el proceso de contratación en el sector sanitario deja ver cómo la ausencia de condiciones enmarcadas dentro un trabajo decente(80) -que implique una adecuada forma de contratación, salario justo, horas de trabajo apropiadas y condiciones del trabajo favorables-, propician una inadecuada prestación del servicio, y con ello, el aumento de rotación de personal, siendo este un fenómeno creciente, especialmente, en aquellas empresas que tienen como misión la prestación de servicios de salud(81).

Aún más, usualmente, el factor más común es la baja remuneración salarial con respecto a la funciones y responsabilidades que se cumplen; lo anterior, afecta de manera negativa a las organizaciones, debido al aumento de los gastos y costos que se generan, aunado a que esta problemática puede llevar a la presencia de accidentes de trabajo, enfermedades laborales, además de dificultades económicas, sociales, familiares, entre otras( ${ }^{(81)}$.

Como se ha mencionado, se considera imprescindible el talento humano en las organizaciones, por ello, la gestión adecuada es fundamental para el desempeño de las mismas y del sistema de salud en conjunto, es 
decir, es gracias al talento humano que se alcanzan los objetivos y metas de los sistemas de salud(82).

Por lo anterior, atraer perfiles idóneos para desempeñar los cargos es fundamental, y es aquí cuando se dialoga de la relevancia del proceso de selección, medular dentro de la gestión humana, ya que permite a las organizaciones integrarse con personas con competencias y perfiles necesarios para lograr los objetivos estratégicos y planes operativos $^{(83)}$.

Es decir, lo anterior, permite contar con un perfil de cargo, identificar requerimientos académicos y de experiencia, con unas competencias requeridas para el desempeño adecuado y relacionarse con el proceso de inducción, el cual, aporta en el conocimiento claro de funciones y roles a asumir(82).

Por otra parte, el proceso de capacitación, el cual favorece el desarrollo y perfeccionamiento de competencias y habilidades, y que, acompañado de los procesos de formación y desarrollo permite la actualización, aprendizaje permanente y aporta al personal sanitario en la asimilación de políticas, protocolos y procedimientos.

Lo anterior, fundamenta el hecho de que se ha generado gran interés en el talento humano, en el conocimiento, habilidades y competencias que posee, llegando con ello a implementar los procesos de capacitación y desarrollo como herramienta para el funcionamiento adecuado de una organización en salud; además, siendo un recurso favorable para la prevención y reducción de accidentes laborales, propiciador de bienestar y del aumento de productividad en la organización(84).

Finalmente, el seguimiento como una forma de acompañar la gestión del desempeño, permite la valoración de la actuación de los colaboradores en el marco de los procedimientos, además, la revisión facilita la generación de estrategias de verificación constante para la prevención de los $\mathrm{EA}^{(5)}$, teniendo presente que, la aparición de estos en la atención en salud, se caracterizan por la complejidad, que se traduce en la cantidad de factores que intervienen en la aparición, como se ha mencionado anteriormente, por las complejidades del procedimiento, el diseño del proceso de atención en salud, condiciones humanas individuales de quienes tienen contacto con el paciente, y la tecnología a utilizar.
Por lo anterior, es necesario referir que no debe ser suficiente para las instituciones de salud pensar en una atención de buena calidad, sin evidenciar efectivamente que es así, y para ello, es prioritario hacer un seguimiento de estos procesos de manera planificada, periódica y sistemática.

En consecuencia, la ausencia de una gestión del talento humano o su inadecuado desarrollo, aporta en la generación de EH que conllevan a los actos inseguros, a la falta de adhesión a protocolos, evidenciándose con ello, la presencia de EA, lo cual, no favorece el cumplimento de los estándares del cuidado asistencial; por lo anterior, es necesario trabajar desde la cultura organizacional, las competencias personales y laborales que coadyuven al reporte de EA, el mejoramiento en el diligenciamiento de la historia clínica, la correcta administración de medicamentos, y en especial, reconocer al ser humano que trabaja.

\section{Limitaciones}

La ausencia de información proveniente de fuentes escritas en idioma inglés, puede ser un limitante al momento de obtener un panorama más amplio frente a los factores relacionados con la ocurrencia de EA.

Por otra parte, las bases de datos de cobro por suscripción impiden el acceso libre a la información, lo que limita los resultados de búsqueda, y con ello, se excluyen documentos que pueden ser importantes para la investigación.

Asimismo, se encontraron estudios llevados a cabo en América Latina, pero fueron publicados en bases de datos específicas de países europeos, por tanto, no se consideraron para la revisión.

\section{Conclusiones}

Los EA son fenómenos complejos que afectan el sistema de salud, sus causas se relacionan con la aparición de múltiples factores, estos, tienen que ver tanto con la organización como con el desempeño del personal de salud, hasta con el actuar del mismo paciente y su familia.

El impacto negativo de los EA no sólo produce un incremento en los costos de la atención en salud, sino que, pone en riesgo el estado de salud del paciente, asimismo, puede ocasionar secuelas emocionales en el personal sanitario. Lo anterior sugiere una adecuada GTH, efectiva en el bienestar laboral de los 
trabajadores, la calidad del servicio prestado y en la disminución de EA.

Adicionalmente, las estrategias de intervención encaminadas a disminuir la ocurrencia de EA, deben primeramente, comprender esta problemática de forma global, sin desconocer la afectación hacia el personal de salud, identificándose estos como segundas víctimas, para posteriormente, formular acciones que no solo se dirijan al personal sanitario, sino que impliquen a la familia y/o acompañante.

En este sentido, las estrategias de intervención deben articularse con los procesos propios de la GTH, tales como la selección del personal, inducción, capacitación, evaluación de desempeño, entre otras, las cuales permitan generar espacios de aprendizaje y herramientas de acción, con las que se pueda mitigar la ocurrencia de EA.

Pues bien, la prevención de los EA es posible y son numerosas las estrategias aplicadas por los diferentes sistemas de salud: estimular el trabajo en equipo, fortalecer la capacitación del personal, estandarizar procedimientos y protocolos, y crear la cultura del reporte voluntario de errores, así como, mejorar las condiciones de trabajo conjuntamente con el tipo de contratación, esto último en relación con la estabilidad laboral.

Finalmente, es necesario intervenir sobre los factores de riesgo psicosocial y analizar condiciones estructurales de los puestos de trabajo, dado que, mejorar las condiciones de trabajo y tipo de contratación, son aspectos que aportan en la salud y el bienestar del trabajador.

Conflicto de intereses: Ninguno declarado por los autores.

\section{Referencias}

1. Organización Mundial de la Salud. Marco Conceptual de la Clasificación Internacional para la Seguridad del Paciente. Informe técnico definitivo. Ginebra [Internet]. 2009 [consultado 2020 Mar 19]. Disponible en: https://www.who.int/patientsafety/implementation/icps/i cps_full_report_es.pdf

2. Molina-Marím G, Oquendo-Lozano T, Rodríguez-Garzón S, Montoya-Gómez N, Vesga-Gómez C, Lagos-Campos N, et al. Gestión del talento humano en salud pública. Un análisis en cinco ciudades colombianas. Gerencia y Políticas de Salud [Internet]. 2016 [consultado 2020 Mar 19];15(30):108-25. DOI: 10.11144/Javeriana.rgyps15-30.gtsp.

3. Daza Ramírez D, Plaza Gómez MT, Hernández Riaño HE. Factores de riesgo intralaboral asociados al clima organizacional y su efecto sobre el síndrome de boreout en el sector salud. Revista Espacios [Internet]. 2017 [consultado 2020 Mar 18];38(58):3. Disponible en: http://www.revistaespacios.com/a17v38n58/17385803.ht $\mathrm{ml}$

4. Organización Mundial de la Salud. Informe sobre la salud en el mundo sobre Reducir los riesgos y promover una vida sana [Internet]. 2002 [consultado 2020 Nov 25]. Disponible en: https://apps.who.int/iris/handle/10665/67455

5. De los Ángeles Páramo M. Factores de Riesgo y Factores de Protección en la Adolescencia: Análisis de Contenido a través de Grupos de Discusión. Terapia psicológica [Internet]. 2011 [consultado 2020 Mar 23];29(1):85-95. DOI: 10.4067/S0718-48082011000100009.

6. Ramírez-Rueda JC, Aroca-Posso YY. Factores de riesgo psicosociales en entidades prestadoras de servicios de salud [Trabajo de grado]. Bucaramanga, Colombia: Universidad Cooperativa de Colombia; 2015 [consultado 2020 Mar 22]. Disponible en: https://repository.ucc.edu.co/bitstream/20.500.12494/30 5/1/Factores\%20de\%20riesgo $\% 20$ psicosociales $\% 20$ en $\% 2$ 0entidades $\% 20$ prestadoras $\% 20 \mathrm{de} \% 20$ servicios $\% 20 \mathrm{de} \% 2$ 0salud.pdf

7. González-Anaya LS, Polo-García C. Riesgos psicosociales y sus efectos en el personal del sector salud en Colombia. Un estudio comparativo [Trabajo de grado]. Bogotá D.C, Colombia: Universidad Sergio Arboleda; 2013 [consultado 2020 Mar 28]. Disponible en: http://hdl.handle.net/11232/890

8. Becerra-Ostos LF, Quintanilla D, Vásquez XA, RestrepoGuerrero HF. Factores determinantes psicosociales asociados a patologías de stress laboral en funcionarios administrativos en una EPS de Bogotá. Revista de Salud Pública [Internet]. 2020 [consultado 2021 Ene 17];20(5):574-78. Disponible en: https://www.scielosp.org/pdf/rsap/2018.v20n5/574578/es

9. Castro-Méndez NP. Riesgos Psicosociales y Salud Laboral en Centros de Salud. Cienc Trab [Internet]. 2018 [citado 2021 Sep 30];20(63):155-9. Disponible en: http://www.scielo.cl/scielo.php?script=sci_arttext\&pid=S0 718-24492018000300155\&lng=es

10. Matabanchoy Tulcán SM. Salud en el trabajo. Universidad y salud [Internet]. 2012 [consultado 2020 Sep 13];1(15):87$102 . \quad$ Disponible en: http://www.scielo.org.co/pdf/reus/v14n1/v14n1a08.pdf

11. Ferreira Umpiérrez $A$, Fort Fort $Z$, Chimielli Tomás V. Adverse events in health and nursing care: patient safety from the standpoint of the professional's experience. Texto $y$ Contexto -Enfermagem [Internet]. 2016 [consultado 2020 May 22];24(2):310-5. DOI: 10.1590/010407072015000122014.

12. Estrada González C, González Restrepo LD. Sucesos adversos relacionados con el acto quirúrgico en una institución de salud de tercer nivel. Revista Cubana de Salud Pública [Internet]. 2018 [consultado 2020 Abr 03];44(3):e998. Disponible en: https://www.medigraphic.com/pdfs/revcubsalpub/csp2018/csp183g.pdf

13. Ministerio de salud. Seguridad del paciente y atención segura paquetes instruccionales. Paquetes Instruccionales: Guía técnica "Buenas prácticas para la seguridad del paciente en la atención en salud" [Internet]. 2014 [citado 2020 Nov 27]. Disponible 
https://www.minsalud.gov.co/sites/rid/Lists/BibliotecaDi gital/RIDE/DE/CA/Guia-buenas-practicas-seguridadpaciente.pdf

14. Wagner A, Rieger MA, Manser T, Sturm H, Hardt J, Martus P, et al. Healthcare professionals' perspectives on working conditions, leadership, and safety climate: a cross-sectional study. BMC health services research [Internet]. 2019 [citado 2021 Sep 27];19(1):53. Disponible en: https://bmchealthservres.biomedcentral.com/articles/10.1 186/s12913-018-3862-7

15. Bautista Rodríguez LM, Parada Rico DA, Ballesteros N, Rodríguez J, Ornella Carreño Z, Guampe Bayona L, et al. Frecuencia, evitabilidad y consecuencias de los eventos adversos. Revista ciencia y cuidado [Internet]. 2011 [consultado 2020 May 26];8(1):75-83. Disponible en: https://dialnet.unirioja.es/servlet/articulo? codigo=385352 3

16. Portela Romero MP, González BR, Calvo Rodríguez MS. Error humano, seguridad del paciente y formación en medicina. Educación Médica [Internet]. 2019 [citado 2021 Sep 30];20(Suppl 1):169-74. DOI: 10.1016/j.edumed.2017.07.027.

17. Zago Novaretti MC, de Vasconcelos Santos E, Quitério LM, Daud-Gallotti RM. Sobrecarga de trabajo de enfermería e incidentes y eventos adversos en pacientes de UCI. Revista Brasileira de Enfermagem [Internet]. 2014 [consultado 2020 Jun 12];67(5):692-9. DOI: 10.1590/0034-7167.2014670504.

18. Amores Agulla T, Marrero Quesada JÁ. Mala praxis médica en el quirófano. Revista Cubana de Cirugía [Internet]. 2015 [consultado 2020 Jun 20];54(2):187-94. Disponible en: http://scielo.sld.cu/pdf/cir/v54n2/cir12215.pdf

19. Barrientos-Sánchez J, Hernández-Zavala $M$, Zárate-Grajales RA. Factores relacionados con la seguridad y la calidad en la atención del paciente pediátrico hospitalizado. Enfermería Universitaria [Internet]. 2019 [consultado 2020 Jun 20];16(1):52. DOI: 10.22201/eneo.23958421e.2019.1.592.

20. Machado Duarte S, Azevedo Queiroz AB, Büscher A, Conceição Stipp MA. El error humano en el cotidiano de la atención de enfermería en terapia intensiva. Rev Latino-Am Enfermagem [Internet]. 2015 [consultado $2020 \mathrm{Abr}$ 15];26(6):1074-81. DOI: 10.1590/0104-1169.0479.2651.

21. Perestelo Pérez L, Medina Arana V, de Castro Peraza ME, Perdomo Hernandez AM. Valoración crítica de la evidencia, lectura crítica: Revisión sistemática y metanálisis [Internet]. 2014 [consultado 2020 Mar 19]. Disponible en: https://campusvirtual.ull.es/ocw/mod/resource/view.php ?id=6134

22. Moher D, Shamseer L, Clarke M, Ghersi D, Liberatî A, Petticrew M, et al. Preferred reporting items for systematic review and meta-analysis protocols (prisma-p) 2015 statement. Systematic Reviews [Internet]. 2015 [consultado 2020 Mar 19];4(1):1-9. Disponible en: https://systematicreviewsjournal.biomedcentral.com/articl es/10.1186/2046-4053-4-1?report=reader

23. Achury-Saldaña D, Rodríguez-Colmenares S, Díaz-Álvarez JC, Gómez J, Gómez-Hernández A, Díaz JE, et al. Caracterización de los eventos adversos reportados por enfermería en unidades de cuidado intensivo en Bogotá (Colombia). Salud Uninorte [Internet]. 2017 [consultado 2020 Abr 29]33(2):105-17. Disponible en: https://www.redalyc.org/articulo.oa?id=817/8175318900 5

24. Toffoletto MC, Barbosa RL, Andolhe R, Machado de Oliveira E, Janzantte Ducci A, Grillo Padilha K. Fatores relacionados à ocorrência de eventos adversos em pacientes idosos críticos. Revista Brasileira de Enfermagem [Internet]. 2016 [consultado 2021 Sep 25];69(6):977-83. DOI: 10.1590/0034-7167-2016-0199. ISSN 1984-0446.

25. Palacios-Barahona AU, Bareño Silva J. Factores asociados a eventos adversos en pacientes hospitalizados en una entidad de salud en Colombia. Rev CES Med [Internet]. 2012 [consultado 2021 Sep 25];26(1):19-28. Disponible en: http://www.scielo.org.co/pdf/cesm/v26n1/v26n1a03.pdf

26. Vargas Bermúdez Z. Factores personales, laborales y contextuales del profesional de enfermería que inciden en la presencia de un evento adverso. Enfermería Actual en Costa Rica [Internet]. 2016 [consultado 2020 Abr 30];(31). Disponible https://www.redalyc.org/articulo.oa?id=44846315005

27. Zárate-Grajales R, Arreola-Olvera S, Cantoral-Hernández A, Hernández-Corral S, Sánchez-Angeles S, Valdez-Labastida R, et al. Factores relacionados con eventos adversos reportados por enfermería en unidades de cuidados intensivos. Proyecto Multicéntrico. Enfermería Universitaria [Internet]. 2018 [consultado 2020 May 25];12(2):63-72. DOI: 10.1016/j.reu.2015.03.003.

28. Machado Duarte S, Conceição Stipp MA, da Silva MM, de Oliveira FT. Eventos adversos e segurança na assistência de enfermagem. Revista Brasileira de Enfermagem [Internet]. 2015 [consultado 2021 Sep 25];68(1):136-46. DOI: 10.1590/0034-7167.2015680120p.

29. Caselli Pacheco F, Santos Domingues CM, Kalume Maranhão AG, Deotti Carvalho SM, da Silva Teixeira AM, Moreira Braz R, et al. Análise do Sistema de Informação da Vigilância de Eventos Adversos Pós-Vacinação no Brasil, 2014 a 2016. Revista Panamericana de Saúde Pública [Internet]. 2018 [consultado 2020 May 25];42:e12. Disponible en: https://www.scielosp.org/article/rpsp/2018.v42/e12/

30. Carvalho de Oliveira A, García PC, de Souza Nogueira L. Carga de trabajo de enfermería y ocurrencia de eventos adversos en cuidados intensivos: una revisión sistemática. Revista de Escola de Enfermagem da USP [Internet]. 2016 [consultado 2020 May 25];50(4):679-89. DOI: 10.1590/S0080623420160000500020.

31. Da Silva de Paiva MC, Rupp de Paiva SA, Wey Berti H. Eventos adversos: análisis de un instrumento de notificación utilizado en el manejo de enfermería. Revista de la Escuela de Enfermería de la USP [Internet]. 2010 [consultado 2020 May 20];44(2):287-94. Disponible en: https://www.scielo.br/pdf/reeusp/v44n2/07.pdf

32. Pontes Lucena AR, de Oliveira Souza LR, Percio J, Deotti Carvalho SM, Martins Romano AP, Santos Domingues CM. Factores asociados con la gravedad de los eventos adversos tras la vacunación contra la fiebre amarilla durante el mayor brote de la enfermedad registrado en Brasil, 2016-2017. Epidemiología y servicios de salud [Internet]. 2020 [consultado 2020 May 28];29(1):e2018331. DOI: 10.5123/s1679-49742020000100017.

33. Auz Vaca J, Muñoz Navarro P, Flores Grijalba M del C, Altamirano Zabala GN, Meneses Dávila MS, Barahona Cisneros ME, et al. Eventos adversos relacionados con los cuidados de enfermería en el Hospital de Ibarra, provincia de Imbabura. Enfermería Investiga [Internet]. 2016 [consultado 2020 Abr 18];1(3):102-6. Disponible en: https://dialnet.unirioja.es/servlet/articulo? codigo=619425 7

34. De Oliveira Lessa SR, de Melo Bezerra JN, Cristovam Barbosa SM, Oliveira de Andrade G, de Oliveira AK. Prevalência e 
fatores associados para a ocorrência de eventos adversos no serviço de hemodiálise. Texto \& Contexto - Enfermagem [Internet]. 2018 [consultado 2020 May 20];27(3):e3830017. DOI: $10.1590 / 0104-07072018003830017$.

35. Fassini P, Hahn GV. Riscos à segurança do paciente em unidade de internação hospitalar: concepções da equipe de enfermagem. Rev Enferm UFSM [Internet]. 2012 [consultado 2020 Ago 25];2(2):290-9. DOI: 10.5902/217976924966.

36. Ferreira-Umpiérrez AH, Chiminelli-Tomás V. Aspectos significativos quirúrgicos de la experiencia de haber sido responsable de un evento adverso en salud. Aquichan [Internet]. 2014 [consultado 2020 Abr 03];14(3):294-302. Disponible

en: http://www.scielo.org.co/scielo.php?script=sci_arttext\&pid $=$ S1657-59972014000300002

37. Rodríguez-Suárez J, Lamy P, Fajardo-Dolci G, SantacruzVarela J, Hernández-Torres F. Incidentes de seguridad del paciente notificados por 22 hospitales mexicanos, mediante el sistema SIRAIS. Revista CONAMED [Internet]. 2012 [consultado 2020 Mar 15];17(2):52-8. Disponible en: https://www.medigraphic.com/pdfs/conamed/con2012/con122b.pdf

38. Navas-Torres J, Alvarado-Muriel P, González-Cartagena JL, Delgado-Ortiz SA, Domínguez CL, Valero-Cárdenas H, et al. Eventos adversos relacionados con el cuidado de enfermería. Revista Cubana de Enfermería [Internet]. 2014 [consultado 2020 Mar 25];30(1):19-27. Disponible en: http://scielo.sld.cu/pdf/enf/v30n1/enf03114.pdf

39. Jiménez Flores EY, Alayola Sansores A, Mancebo Hernández A, Campos Castolo M. Eventos adversos y burnout en profesionales de una clínica de atención primaria. Revista CONAMED [Internet]. 2018 [consultado 2020 Ago 21];23(2):66-72. Disponible

https://www.medigraphic.com/cgi-

bin/new/resumen.cgi?IDARTICULO=80118

40. Ferreira Arrieche M, Wegner W. Eventos adversos no cuidado da criança: concepções de familiar/cuidador na atenção básica. Ciência \& Saúde [Internet]. 2013 [consultado 2020 May 13];6(3):190-6. DOI: 10.15448/1983652X.2013.3.13471.

41. Fariña D, Rodríguez S, Vaihinger $M$, Salva V, Porto $R$, Ganduglia M, et al. Mortalidad neonatal: análisis causa raíz. Estudio prospectivo de los factores vinculados a la muerte de recién nacidos internados en maternidades seleccionadas del sector público de la república argentina. Revista del Hospital Materno Infantil Ramón Sardá [Internet]. 2012 [consultado 2020 Ago 22];31(3):117-24. Disponible en: https://www.redalyc.org/pdf/912/91225251004.pdf

42. Fajardo-Dolci GE, Rodríguez-Suárez J, Gallegos-Alvarado M, Córdoba-Ávila MÁ, Flores-Bravo MC. Percepción de la frecuencia y causas de los eventos adversos por medicación en enfermería. Revista CONAMED [Internet]. 2009 [consultado 2020 Ago 16];14(1):22-7. Disponible en: https://dialnet.unirioja.es/servlet/articulo?codigo=362821 3. ISSN-e 1405-6704

43. Dos Santos R, Passaura da Luz MA, Borges F, da Silva Carvalho A. Búsqueda activa contribuye a la identificación de eventos adversos e incidentes en unidad de cuidados intensivos. Enfermería Global [Internet]. 2017 [consultado 2020 Mar 27];16(4):465-98. DOI: 10.6018/eglobal.16.4.269601.

44. Parra Pareja CV, Lopéz Rivas JS, Bejarano CH, Puerto Guerrero AH, Galeano Goyes ML. Eventos adversos en un hospital pediátrico de tercer nivel de Bogotá, 2013-2014.
Facultad Nacional de Salud Pública [Internet]. 2017 [consultado 2020 May 21];35(2). Disponible en: https://dialnet.unirioja.es/servlet/articulo? codigo $=586811$ 6

45. Achury Saldaña D, Rodríguez SM, Díaz JC, Cavallo E, Zarate Grajales R, Vargas Tolosa R, et al. Estudio de eventos adversos, factores y periodicidad en pacientes hospitalizados en unidades de cuidado intensivo. Enfermería Global [Internet]. 2016 [consultado 2020 Mar 12];15(42):324-40. Disponible en: http://scielo.isciii.es/scielo.php?script=sci_arttext\&pid=S16 95-61412016000200011\&lng=es\&tlng=es

46. Zago Novaretti MC. Aplicación del análisis de causa raíz como herramienta en la gestión de seguridad hospitalaria. Revista de Administración de la UFSM [Internet]. 2014 [consultado 2020 Ago 25];7(3):442-52. DOI: 10.5902/1983465916272.

47. Braz Pavão AL, Andrade D, Mendes W, Martins M, Travassos C. Estudio de la incidencia de eventos hospitalarios adversos, Río de Janeiro, Brasil: evaluación de la calidad de la historia clínica del paciente. Revista Brasileira de Epidemiologia [Internet]. 2011 [consultado 2020 Mar 20];14(4):651-61. Disponible en: https://www.scielo.br/j/rbepid/a/tF95SQRXHW7TGTQ5Js $\mathrm{kbqpG} /$ ?format=pdf\&lang=pt

48. Lopes de Figueiredo M, D'Innocenzo M. Eventos adversos relacionados con las prácticas asistenciales: una revisión integradora. Enfermería Global [Internet]. 2017 [consultado 2020 Ago 22];16(3):605-20. Disponible en: https://scielo.isciii.es/pdf/eg/v16n47/1695-6141-eg-1647-00605.pdf

49. Estebanez Roque K, Tonini T, Caetano Prates E. Eventos adversos en la unidad de cuidados intensivos: impacto en la mortalidad y la duración de la estancia en un estudio prospectivo. Cadernos de Saúde Pública [Internet]. 2016 [consultado 2020 Abr 12];32(10):e00081815. DOI: 10.1590/0102-311X00081815.

50. Echevarría Zuno S, Sandoval Castellanos F, Gutiérrez Dorantes S, Alcantar Bautista A, Cote Estrada L. Eventos adversos en cirugía. Cirujano general [Internet]. 2011 [consultado 2020 Nov 05];33(3):163-9. Disponible en: http://www.scielo.org.mx/scielo.php?script=sci_arttext\&pi $\mathrm{d}=$ S1405-00992011000300005\&lng=es\&tlng=es

51. Spironello RA, Nakamura Cuman RK. Caracterización de eventos adversos en una unidad de cuidado intensivo neonatal. Revista científica de enfermería-RECIEN [Internet]. 2019 [consultado 2020 Ago 22];9(28):131-6. Disponible en: https://recien.com.br/index.php/Recien/article/view/324

52. Álvarez-Maldonado P, Hernández-Ríos G, Reding-Bernal A, Cicero-Sabido R. Eventos adversos en una nueva unidad de cuidados intensivos. Influencia del diseño y la tecnología de las instalaciones en las tasas de incidencia. Gaceta medica de Mexico [Internet]. 2019 [consultado 2020 Mar 15];155(6):613-8. DOI: 10.24875/gmm.19005421.

53. Rendón Román MC, Restrepo Franco SV, García Castañeda JJ. Determinación de la frecuencia, causas y evitabilidad de los eventos adversos en el servicio de pediatría de un IPS Quindio 2014. Journal of Research of the University of Quindío [Internet]. 2016 [consultado 2020 Ago 25];28(1):43-55. DOI: 10.33975/riuq.vol28n1.34.

54. Poblete Umanzor R, Conejeros Fritz S, Corrales Fernández MJ, Miralles Bueno JJ, Aranaz J. Revisión sistemática de la literatura acerca de la seguridad de los pacientes hospitalizados en servicios médicos. Revista de Calidad 
Asistencial [Internet]. 2011 [consultado 2021 Sep 23];26(6):359-66. DOI: 10.1016/j.cali.2011.09.005.

55. Fondo de Investigaciones Sanitarias, Fondos FEDER, la Conselleria d'Educació, Investigació, Cultura i Esport (Generalitat Valenciana) y la Fundación para el Fomento de la Investigación Sanitaria y Biomédica de la Comunitat Valenciana (FISABIO). Proyecto de investigación: Segundas y terceras víctimas [Internet]. 2019 [consultado 2020 Nov 30]. Disponible en: http://www.segundasvictimas.es/

56. Torijano-Casalengua ML, Astier-Peña P, Mira-Solves JJ. El impacto que tienen los eventos adversos sobre los profesionales sanitarios de atención primaria y sus instituciones. Atención Primaria [Internet]. 2016 [consultado 2020 May 9];48(3):143-6. DOI: 10.1016/j.aprim.2016.01.002.

57. Gámez-Molina YD, Guarnizo-Lima M de J, Guarguati-Ramos AD. Eventos adversos relacionados con infecciones intrahospitalarias: una revisión sistemática [Trabajo de grado]. Santa Marta, Colombia: Universidad Cooperativa de Colombia; 2018 [consultado 2021 Sep 23]. Disponible en: https://repository.ucc.edu.co/bitstream/20.500.12494/52 16/1/REVISION\%20NARRATIVA\%20DE\%20EVENTOS\%20 ADVERSOS\%20ASOCIADOS\%20A\%20INFECCIONES\%20IN TRAHOSPITALARIAS.pdf

58. Carrillo I, Mira JJ, Astier Peña MP, Pérez-Pérez P, CaroMendivelso J, Olivera G, et al. Eventos adversos evitables en atención primaria. Estudio retrospectivo de cohortes para determinar su frecuencia y gravedad. Atención Primaria [Internet]. 2020 [consultado 2020 Nov 26];52(10):705-11. DOI: 10.1016/j.aprim.2020.02.008.

59. Vega M, Nuñez G. Experiencias Adversas en la Infancia: Revisión de su impacto en niños de 0 a 5 años. Enfermería Universitaria [Internet]. 2017 [consultado $2020 \mathrm{Abr}$ 6];14(2):124-30. DOI: 10.1016/j.reu.2017.02.004.

60. Mena G, Montané E, Rodríguez M, Beroiz P, López-Núñez JJ, Ballester M. Caracterización y eventos adversos relacionados con la asistencia sanitaria en pacientes infectados por el SARS-CoV-2 fallecidos en un hospital de tercer nivel. Medicina Clínica [Internet]. 2021 [citado 2021 Sep 30]; 156(6):277-80. DOI: 10.1016/j.medcli.2020.11.002.

61. Rosas Aparcana KG. Factores asociados a los eventos adversos en centro quirúrgico [Trabajo de grado]. 2017 [citado 2021 Sep 30]. Disponible en: http://repositorio.uwiener.edu.pe/handle/123456789/140 4

62. Homs-Romero È, Esperón Güimil J, Loureiro Rodríguez MT, Cegri Lombardo F, Cueto Pérez M, Paniagua Asensio ML, et al. Percepción de los profesionales sanitarios sobre la gravedad de las úlceras por presión como evento adverso. Gerokomos [Internet]. 2018 [citado 2021 Sep 30];29(1):39-44. Disponible

en: http://scielo.isciii.es/scielo.php?script=sci_arttext\&pid=S11 34-928X2018000100039\&lng=es

63. Mira JJ, Ferrús L, Silvestre C, Olivera G. Qué, quién, cuándo, dónde y cómo informar a los pacientes tras un evento adverso: un estudio cualitativo. Enfermería Clínica [Internet]. 2017 [citado 2021 Sep 30];27(2):87-93. Disponible en: https://www.sciencedirect.com/science/article/abs/pii/S1 13086211630033X

64. Quispe Casanova AA. Conocimientos y actitudes sobre eventos adversos en la cultura de seguridad del paciente del personal de salud de la Clínica Divino Niño Jesús. Lima. 2019 [Tesis de maestria]. Lima, Perú: Universidad Norbert
Wiener; 2020 [citado 2021 Sep 30]. Disponible en: http://repositorio.uwiener.edu.pe/bitstream/handle/1234 56789/4039/T061_43277477_M.pdf?sequence=1

65. Pasto Salud E.S.E. Paquete instruccional. Ilustrar al paciente acerca del autocuidado de su seguridad Pasto Salud E.S.E. Versión 2.0. Colombia [Internet]. 2017 [consultado 2020 Oct 03]. Disponible en: https://www.pastosaludese.gov.co/images/planeacion/20 17/PAQUETEINSTRUCCIONALAUTOCUIDADOUSUARIOSES T.pdf

66. Organización Panamericana de la Salud, Organización Mundial de la Salud. Boletín CONAMED-OPS. Órgano de difusión del Centro colaborador en materia de Calidad y Seguridad del Paciente. Los Eventos Adversos y la Seguridad del Paciente [Internet]. 2015 [consultado 2020 Oct 08]. Disponible

en: http://www.conamed.gob.mx/gobmx/boletin/pdf/boletin3 /eventos_adversos.pdf

67. Zárate-Grajales RA, Salcedo-Álvarez RA, Olvera-Arreola SS, Hernández-Corral S, Barrientos-Sánchez J, Pérez-López MT, et al. Eventos adversos en pacientes hospitalizados reportados por enfermería: un estudio multicéntrico en México. Enfermería Universitaria [Internet]. 2017 [consultado 2020 Jul 25];14(4):277-85. DOI: 10.1016/j.reu.2017.08.005.

68. Ministerio de salud de Colombia. Guía técnica: Buenas prácticas para la seguridad del paciente en la atención en salud. Evaluación de la frecuencia de eventos adversos y monitoreo de aspectos claves relacionados con la seguridad del paciente [Internet]. 2015 [consultado 2020 Nov 30]. Disponible en: https://www.minsalud.gov.co/sites/rid/Lists/BibliotecaDi gital/RIDE/DE/CA/Evaluar-frecuencia-eventosadversos.pdf

69. Ite Vargas JC. Caracterización de la infraestructura en salud de la región pacífico de Colombia (Proyecto de grado). Santiago de Cali: Universidad Autónoma de Occidente [Internet]; 2019 [consultado 2020 Ago 25]. Disponible en: https://red.uao.edu.co/bitstream/handle/10614/11800/T 08949.pdf;jsessionid=47E6D4CE46C344C187E776DF5086 10BA?sequence $=5$

70. Vargas-Cruz LD, Coral-Ibarra R del C, Barreto-Osorio RV. Carga mental en personal de enfermería: Una revisión integradora. Revista Ciencia y Cuidado [Internet]. 2020 [citado 2021 Sep 30];17(3):108-21. Disponible en: https://dialnet.unirioja.es/servlet/articulo?codigo=773235 6

71. Rocco C, Garrido A. Seguridad del paciente y cultura de seguridad. Revista Médica Clínica Las Condes [Internet]. 2017 [citado 2021 Sep 30];28(5):785-95. Disponible en: https://www.sciencedirect.com/science/article/pii/S0716 864017301268

72. Granero Lázaro A. Calidad de vida laboral de las enfermeras: Evaluación y propuestas de mejora [Tesis Doctoral].

Barcelona, España: Universidad de Barcelona; 2017 [citado 2021 Sep 30]. Disponible en: http://diposit.ub.edu/dspace/bitstream/2445/113788/1/ AGL_TESIS.pdf

73. Sanz-Gómez AI, Iriarte-Ramos S, Gascón-Catalán A. Aspectos sociodemográficos y laborales en el error asistencial de enfermería. Enfermería Global [Internet]. 2016 [consultado 2020 Ago 25];15(3):176-87. DOI: 10.6018/eglobal.15.3.214021. 
74. Ministerio de Sanidad, Servicios Sociales e Igualdad. La prevención de los eventos adversos. La cultura de la seguridad. Prácticas Seguras [Internet]. España; 2019 [citado 2020 Nov 20]. Disponible en: https://cursos.seguridaddelpaciente.es/courses/cur002/0 7/01-contenidos.pdf

75. Da Silva de Paiva MC, Popim R, Melleiro MM, Rizzato Tronchim DM, Molina Lima SA, Monti Juliani CM. The reasons of the nursing staff to notify adverse events. Rev Latino-Am Enfermagem [Internet]. 2014 [consultado 2020 Ago 25];22(5):747-54. Disponible en: https://www.scielo.br/pdf/rlae/v22n5/es_0104-1169rlae-22-05-00747.pdf

76. Faria Rocha R de P, Moura Pinho DL. Ocurrencia de eventos adversos en unidades públicas de hemodiálisis. Enf Global [Internet]. 2019 [citado 2021 Sep 30];18(3):1-34. Disponible en: https://revistas.um.es/eglobal/article/view/343361

77. Peña M, Melleiro MM. Eventos adversos resultantes de fallas de comunicación: reflexiones sobre un modelo para la transición de la atención. UFSM Nursing Journal [Internet]. 2018 [consultado 2020 Ago 20];8(3):616-25. DOI: $10.5902 / 2179769225432$.

78. Soto Aranda LK. Gestión del Talento humano y cultura de la seguridad del paciente en el laboratorio en un hospital público, el Agustino, 2018 [Tesis de grado]. Lima, Perú: Universidad Cesar Vallejo; 2019 [consultado 2020 May 02]. Disponible en: https://repositorio.ucv.edu.pe/bitstream/handle/20.500.1 2692/34153/Soto_AL.pdf?sequence=1

79. Saravia Yaya RB. Cultura de seguridad del paciente y gestión del talento humano en el personal de enfermería en áreas críticas del Hospital Nacional Dos de Mayo, Lima-2017 [Tesis de Grado]. Perú: Universidad Cesar Vallejo; 2018 [citado 2021 Sep 30]. Disponible en: https://core.ac.uk/download/pdf/225589714.pdf

80. Organización Internacional del Trabajo (OIT). 23 Condiciones del Trabajo [Internet]. 2016 [citado 2020 Nov 20]. Disponible en: https://www.ilo.org/global/topics/dw4sd/themes/workin g-conditions/lang--es/index.htm

81. Rueda Mahecha YM, Silva Giraldo CA, Suarez Suarez DA. La gestión del talento humano en las instituciones prestadoras de salud IPS. Revista ITEES [Internet]. 2020 [consultado 2021 Ene 12];3(3):179-96. DOI: 10.34893/itees.v3i3.58.

82. Torres-Flórez D, Godoy-González B, Gallardo-Lichaá N. Procesos de reclutamiento y selección en organizaciones de salud: Caso Villavicencio-Colombia. Desarrollo Gerencial [Internet]. 2019 [consultado 2020 Mar 11];11(1):60-78. DOI: 10.17081/dege.11.1.3380.

83. Sanchez Zambrano IJ. Diseño de un sistema de reclutamiento y selección por competencias para disminuir la rotación de personal en Sepronac. Cía. Ltda. Quito [Tesis de Maestría]. Ecuador: Universidad Andina Simón Bolívar; 2020 [consultado 2020 Sep 03]. Disponible en: https://repositorio.uasb.edu.ec/bitstream/10644/7763/1/ T3352-MDTH-S\%C3\%A1nchez-Dise\%C3\%B1o.pdf

84. Miranda González AD. Análisis del impacto del síndrome de Burnout en el talento humano en salud y la seguridad de los pacientes [Tesis de Grado]. Bogotá D.C, Colombia: Universidad Militar de Nueva Granada; 2018 [consultado 2020 Sep 30]. Disponible en: https://repository.unimilitar.edu.co/handle/10654/18188 